\title{
RDUS
}

Revue de DROIT UNIVERSITÉ DE SHERBROOKE

Titre : $\quad$ LA RESPONSABILITÉ CIVILE DES SAGES-FEMMES

Auteur(s) : $\quad$ François TÔTH

Revue: $\quad$ RDUS, 2005-2006, volume 36, numéro 1-2

Pages: $\quad 1-43$

ISSN : $\quad 0317-9656$

Éditeur : Université de Sherbrooke. Faculté de droit.

URI : $\quad$ http://hdl.handle.net/11143/11874

DOI : $\quad$ https://doi.org/10.17118/11143/11874 
Page vide laissée intentionnellement. 


\section{ARTICLE \\ LA RESPONSABILITÉ CIVILE DES SAGES-FEMMES}

par François TÔTH*

La profession de sage-femme au Québec a été officiellement reconnue le 19 juin 1999 par l'adoption de la Loi sur les sages-femmes ${ }^{l}$. La sage-femme a obtenu le statut de professionnelle de la santé et l'Ordre professionnel des sages-femmes du Québec a été créé. Depuis le 10 juin 2004, la sage-femme peut pratiquer un accouchement à domicile. Quel long parcours depuis l'établissement des premières maisons de naissance dans le cadre des projets-pilotes! Cette nouvelle profession et un cadre juridique particulier interpellent le juriste. Quels sont les droits et obligations de cette nouvelle intervenante en périnatalité? Et surtout, quel est le cadre d'analyse de sa responsabilité civile professionnelle?

In Quebec, the practice of midwifery was recognized as a profession the $19^{\text {th }}$ of June, 1999 following adoption of the Midwives Act ${ }^{2}$. Midwives now enjoy the status of health professionals and a new professional association has been created, the Ordre professionnel des sages-femmes du Québec. Since the $10^{\text {th }}$ of June, 2004, midwives are now allowed to perform home births. This constitutes a significant development since the establishment of the first birthing homes during the pilot projects in the 90's. As a «new» health profession, the legal status of midwifery requires some analysis, especially as regards the rights and obligations of the midwife and more importantly, the extent of a midwife's civil liability

*. Avocat et professeur à la Faculté de droit de l'Université de Sherbrooke.

1. L.R.Q. c. S-0.1.

2. Ibid. 


\section{SOMMAIRE}

La sage-femme : nouvelle professionnelle en périnatalité $\ldots \ldots \ldots \ldots 3$

1. Cadre légal et réglementaire $\ldots \ldots \ldots \ldots \ldots \ldots \ldots \ldots$

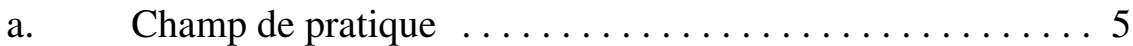

b. Exercice de la pratique de la sage-femme .........6 6

c. Contrôle de la pratique de la sage-femme ..........9

i. Contrôle de l'acte par l'Ordre des sages-femmes

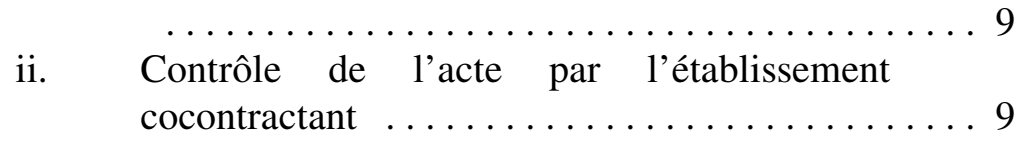

2. Responsabilité civile de la sage-femme $\ldots \ldots \ldots \ldots \ldots \ldots 11$

a. Lien juridique sage-femme/parturiente . . . . . . . . 13

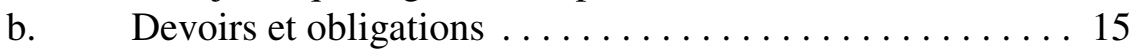

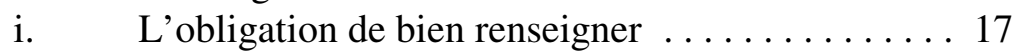

ii. L'obligation de bien soigner . . . . . . . . . 22

iii. L'obligation de suivre ............. 30

3. Pluralité des intervenants et responsabilité plurale $\ldots \ldots \ldots 33$

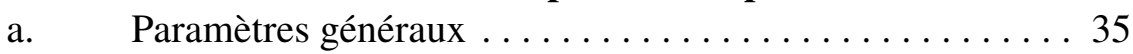

b. Paramètres quant à la sage-femme $\ldots \ldots \ldots \ldots \ldots \ldots$

c. Paramètres quant au lieu de naissance $\ldots \ldots \ldots \ldots \ldots 36$

d. Paramètres quant aux risques obstétricaux . . . . . . . . . 39

i. La sage-femme poursuit le suivi de la grossesse

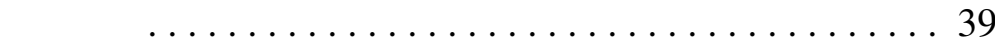

ii. Il y a suivi conjoint sage-femme/médecin . . . . . 40

iii. Il y a transfert de la responsabilité clinique . . . . . 40

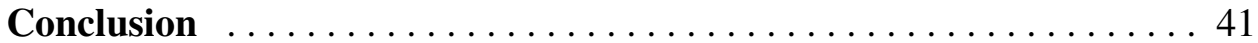

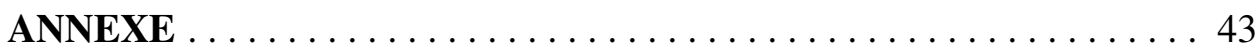




\section{La sage-femme : nouvelle professionnelle en périnatalité}

L'Ordre professionnel des sages-femmes ${ }^{3}$ est l'un des 45 ordres professionnels québécois. Fait remarquable et indice de l'importance de la santé dans la vie et le bien-être des citoyens québécois, plus de la moitié des ordres professionnels concernent la santé dans son acception moderne.

Ce n'est pas notre intention de faire l'historique de la création de cet ordre professionnel au Québec. Nous référons le lecteur aux nombreux articles et ouvrages sur le sujet ${ }^{4}$. Beaucoup a été écrit sur ce symbole de la lutte des femmes pour l'obtention des services de sages-femmes. Ce fut un débat très politisé qui n'est peut-être pas terminé. L'avenir nous le dira.

Ce qu'il faut savoir cependant c'est que la légalisation de la profession a été l'objet d'un long processus de mise à l'essai et d'évaluation dans le cadre des projets-pilotes de 1990 à $1998^{5}$. Cette stratégie des petits pas, même si elle n'allait pas assez vite au goût de certains, s'est avérée fructueuse car elle a pu vaincre les résistances, parfois fondées sur des impressions plutôt que sur des données scientifiques rigoureuses ${ }^{6}$.

3. Ordre des sages-femmes du Québec, voir en ligne : <http://www.osfq.org/> [osfq].

4. Par exemple, Cosmina Caslariu, «La responsabilité civile des sages-femmes au Québec» (1998) 12 R.J.E.U.L. 85, particulièrement aux pages 88 à 93 et notes y citées. La meilleure étude sur la responsabilité civile de la sage-femme québécoise demeure l'article de Me Simard : Caroline Simard, «La responsabilité civile pour la faute de la sage-femme : des projets-pilotes à la légalisation» (2001) 32 R.D.U.S. 59 [Caroline Simard], à l'origine présentée comme essai pour l'obtention de la maîtrise en droit de la santé. L'auteure s'est mérité la bourse d'excellence Chénier-Picard en juin 2000 pour la qualité de son essai.

5. Loi sur la pratique des sages-femmes dans le cadre de projets-pilotes, L.R.Q. c. P-16.1. Loi prolongeant l'effet de certaines dispositions de la Loi sur la pratique des sages-femmes dans le cadre de projets-pilotes, L.Q. 1998, c. 26. Ailleurs au Canada : Alberta : Health Disciplines Act, R.S.A. 2000, c. H-2; Colombie-Britannique : Health Professions Act, R.S.B.C. 1996, c. 183; Manitoba : Midwifery Act, C.C.S.M. c. M125; Ontario : Midwifery Act, 1991, S.O. 1991, c. 31; Terre-Neuve : Midwifery Act, R.S.N.L. 1990, c. M-11.

6. Voir Conseil d'évaluation des technologies de la santé, Les mortinaissances dans le cadre des projets-pilotes de la pratique des sages-femmes au Québec (Rapport) Montréal, CETS, 1999, en ligne: Agence d'évaluation des technologies et des modes d'intervention en santé $<$ http://www.aetmis.gouv.qc.ca>. Voir Jean-François Bégin, «Huit morts évitables» La Presse (26 août 1999) A-8. 
Depuis 1999, la sage-femme est une professionnelle intégrée au système de santé périnatale.

\section{Cadre légal et réglementaire}

La profession de sage-femme est une profession d'exercice exclusif au même titre que l'exercice de la médecine ${ }^{7}$, ce qui signifie que la praticienne doit être titulaire d'un permis délivré par l'Ordre pour porter le titre de sage-femme ${ }^{8}$ et poser les actes professionnels propres à son champ de pratique. La principale fonction d'un ordre professionnel est de s'assurer la protection du public, notamment par le contrôle de l'exercice de la profession par ses membres ${ }^{9}$. La reconnaissance légale de la profession de sage-femme atteste que l'État a considéré que les services de sages-femmes ne pouvaient être dispensés que par des personnes possédant la formation et les qualifications requises par l'Ordre ${ }^{10}$.

Il faut savoir qu'une formation universitaire de 4 ans (130 crédits) est offerte depuis septembre 1999 en pratique sage-femme à l'Université du Québec à Trois-Rivières ${ }^{11}$. Quant aux sages-femmes qui pratiquaient auparavant dans le cadre des projets-pilotes ${ }^{12}$, des dispositions transitoires d'accès à la profession sont prévues ${ }^{13}$.

7. $\quad$ Code des professions, L.R.Q. c. C-26, art. 32, ann. I aux para 1-21.4.

8. Selon l'art. 52 de la Loi sur les sages-femmes, supra note 1, la personne qui, le 30 juin 1999 , est titulaire d'une reconnaissance d'aptitude à pratiquer à titre de sage-femme dans les projets-pilotes, délivrée par le comité d'admission à la pratique des sages-femmes conformément à la Loi sur la pratique des sages-femmes dans le cadre de projets-pilotes, supra note 5, devient également titulaire d'un permis d'exercice de la profession de sage-femme délivré par le Bureau de l'Ordre.

9. $\quad$ Code des professions, supra note 7, art. 23.

10. Code des professions, supra note 7, art. 26.

11. Voir en ligne : Université du Québec à Trois-Rivières <http://www.uqtr.ca/> .

12. Soit les sages-femmes autodidactes et formées à l'étranger qui avaient satisfait au Règlement sur les critères généraux de compétence et de formation des sages-femmes dans le cadre de projets-pilotes, R.R.Q. 1981, c. P-16.1, r. 0.1.

13. Loi sur les sages-femmes, supra note 1, art. 52 et $\mathrm{s}$. 


\section{a. Champ de pratique}

La Loi sur les sages-femmes a défini de façon précise le champ d'activité professionnelle de la sage-femme. Selon l'article 6 de cette loi, constitue l'exercice de la profession de sage-femme tout acte ayant pour objet, lorsque tout se déroule normalement, de donner à une femme les soins et les services professionnels requis pendant la grossesse, le travail et l'accouchement et de donner à une femme et à son enfant les soins et les services professionnels requis durant les six premières semaines de la période postnatale. Ces soins et services professionnels consistent :

$1^{\circ}$ à surveiller et à évaluer la grossesse, le travail, l'accouchement et, durant les six premières semaines, la période postnatale par l'application de mesures préventives et par le dépistage de conditions anormales chez la femme ou son enfant;

$2^{\circ}$ à pratiquer l'accouchement spontané;

$3^{\circ}$ à pratiquer une amniotomie, une épisiotomie et sa réparation ainsi qu'une réparation d'une lacération ou d'une déchirure du premier ou du deuxième degré du périnée. [nos soulignés]

La pratique de la sage-femme s'adresse donc à la grossesse et à l'accouchement qui se déroulent normalement. Un pouvoir de prescrire certains médicaments, examens et analyses liés au champ de pratique est également prévu à la $1 \mathrm{l}^{14}$. De plus, 1 'article 6 n'interdit pas aux infirmières de donner à une femme et à son enfant les soins infirmiers requis pendant la grossesse, le travail, l'accouchement et la période postnatale ${ }^{15}$.

L'exercice de la médecine est, quant à lui, défini comme suit :

31. L'exercice de la médecine consiste à évaluer et à diagnostiquer toute déficience de la santé de l'être humain, à prévenir et à traiter les maladies dans le but de maintenir la santé ou de la rétablir.

14. Loi sur les sages-femmes, supra note 1, art. 8, 59.

15. Ibid., art. 12. 
Dans le cadre de l'exercice de la médecine, les activités réservées au médecin sont les suivantes :

$1^{\circ}$ diagnostiquer les maladies;

$2^{\circ}$ prescrire les examens diagnostiques;

$3^{\circ}$ utiliser les techniques diagnostiques invasives ou présentant des risques de préjudice;

$4^{\circ}$ déterminer le traitement médical;

$5^{\circ}$ prescrire les médicaments et les autres substances;

$6^{\circ}$ prescrire les traitements;

$7^{\circ}$ utiliser les techniques ou appliquer les traitements, invasifs ou présentant des risques de préjudice, incluant les interventions esthétiques;

$8^{\circ}$ exercer une surveillance clinique de la condition des personnes malades dont l'état de santé présente des risques;

$9^{\circ}$ effectuer le suivi de la grossesse et pratiquer les accouchements;

$10^{\circ}$ décider de l'utilisation des mesures de contention. [nos soulignés $]^{16}$

On voit donc les rôles complémentaires de la sage-femme et du médecin. Rien n'empêche l'un et l'autre d'effectuer le suivi d'une grossesse et de pratiquer des accouchements, mais la pratique de la sage-femme est restreinte à la grossesse et à l'accouchement lorsque que tout se déroule normalement. Nous reviendrons sur ce terme.

\section{b. Exercice de la pratique de la sage-femme}

Une sage-femme qui désire exercer sa profession pour un établissement qui exploite un CLSC doit conclure avec lui un contrat de services d'une durée maximale de trois ans ${ }^{17}$. Cette disposition a le mérite d'être claire. La sage-femme est une professionnelle autonome qui ne devient pas la préposée de

16. Loi médicale, L.R.Q. c. M-9, art. 31.

17. Loi sur les services de santé et les services sociaux L.R.Q. c. S-4.2, art. 259.2, 530.78 .1 [LSSSS]. Sous le régime des projets-pilotes, la sage-femme était une employée du CLSC. Changeant de statut, elle doit maintenant conclure un contrat de services avec un CLSC, Loi sur les sages-femmes, art. 67. Voir Caroline Simard, supra note 4, aux pp. 76-77. 
l'établissement ${ }^{18}$, lien de préposition et contrat de services étant antinomiques ${ }^{19}$. Si la demande de la sage-femme est acceptée et si un contrat de services est conclu, le CLSC devra prévoir les droits et les obligations de la sage-femme rattachés à l'exercice de sa profession pour l'établissement ${ }^{20}$. Dans une poursuite en responsabilité civile, l'examen de ce contrat peut être utile afin de déterminer certains devoirs de la sage-femme envers ses patientes, notamment en matière de suivi, de remplacement et de garde.

Il apparaît possible juridiquement pour une sage-femme d'exercer sa profession seule ou avec d'autres sages-femmes sans être rattachée à un CLSC. Toutefois, elle se priverait de l'accès à la maison de naissance ainsi que des services de support médical. Plus concrètement, il pourrait s'agir d'une catégorie de risque qu'un assureur responsabilité ne voudrait pas couvrir ${ }^{21}$.

Comme professionnelle, la sage-femme doit détenir une assurance-responsabilité civile ${ }^{22}$. Les services de sages-femmes sont des services assurés selon la loi et donc disponibles gratuitement au détenteur de la carte d'assurance maladie. Rien ne restreint le droit d'une femme d'accoucher où elle le désire : dans une maison de naissance, un centre hospitalier ou à domicile.

Le concept de la maison de naissance ${ }^{23}$ a été élaboré à l'occasion des projets-pilotes. Le mot le dit : il s'agit d'un endroit entièrement dédié au suivi des grossesses et aux accouchements, le tout dans une atmosphère chaleureuse et familiale. La maison de naissance est rattachée à un centre de santé ${ }^{24}$. Afin de permettre le respect de la réglementation en matière de consultation médicale

18. Malgré le pouvoir disciplinaire du conseil d'administration de l'établissement cocontractant, LSSSS, supra note 17, art. 259.6, 236.

19. C.c.Q., art. 2099.

20. LSSSS, supra note 17, art. 259.5.

21. Le ministre de la santé peut conclure avec l'association professionnelle des sages-femmes des ententes pour l'application des contrats de services sage-femme-CLSC et prévoyant la rémunération des sages-femmes et le remboursement, à l'instar des médecins, de tout ou partie d'une prime d'assurance. LSSSS, supra note 17, art. 432.1.

22. Ibid., art. 259.9. Actuellement, les sages-femmes sont assurées par le Fonds d'assurance de l'Association des hôpitaux du Québec.

23. Voir le site web de la Maison de naissance Mimosa, en ligne : La maison de naissance Mimosa <http://www.mimosa.qc.ca/> [Mimosa].

24. $\quad$ LSSSS, supra note 17, art. 92. 
et de transfert, l'établissement dont relève la maison de naissance doit conclure avec un établissement qui exploite un centre hospitalier une entente assurant, lorsque requis, le support médical à une sage-femme de même que les mesures nécessaires afin de procurer à la femme ou à son enfant, en cas de consultation et de transfert, les soins et les services requis par leur état ${ }^{25}$.

L'accouchement en centre hospitalier sous la responsabilité d'une sage-femme est possible. L'établissement qui a conclu un contrat de services avec une sage-femme peut conclure une entente avec un établissement qui exploite un centre hospitalier afin de permettre à la sage-femme d'y pratiquer des accouchements ${ }^{26}$. Rien cependant n'oblige la sage-femme de pratiquer des accouchements en milieu hospitalier. Mais l'entente est nécessaire si la cliente veut accoucher à l'hôpital sous les soins d'une sage-femme. On remarque que l'entente a lieu entre établissements plutôt qu'entre personnes, ce qui facilite la conclusion de telles ententes. Le contenu d'une telle entente est prévu par la loi :

Cette entente doit prévoir les droits et obligations des deux établissements quant à l'utilisation par les sages-femmes des locaux et de l'équipement de l'établissement qui exploite le centre hospitalier, les conditions de collaboration entre, d'une part, les sages-femmes et, d'autre part, les médecins et le personnel infirmier qui exercent leur profession dans le centre hospitalier, les modalités d'admission et de congé que doivent appliquer les sages-femmes à l'égard des femmes et des enfants qui sont sous leur responsabilité ainsi que toute autre modalité administrative nécessaire au bon fonctionnement de l'entente.

[...] [c]ette entente doit également prévoir qu'elle lie tous les médecins visés par les conditions de collaboration prévues au deuxième alinéa. ${ }^{27}$

On remarque cette fois que la collaboration nécessaire entre la sage-femme et le personnel médical et infirmier n'est pas négociable ou soumis à un bon vouloir capricieux.

25. Ibid., art. 259.11.

26. Ibid., art. 259.10.

27. Ibid., art. 259.10, al. 2, 3 . 
La grande nouveauté est certainement la possibilité pour la future maman d'accoucher à domicile. En effet, le Règlement sur les normes de pratique et les conditions d'exercice lors d'accouchements à domicile ${ }^{28}$ est entré en vigueur le 10 juin $2004^{29}$.

\section{c. Contrôle de la pratique de la sage-femme}

Quoique professionnelle indépendante, la sage-femme est soumise à un contrôle de la qualité des actes posés comme tout professionnel québécois.

\section{i. Contrôle de l'acte par l'Ordre des sages-femmes}

À ce niveau, nous retrouvons le contrôle de la compétence et de la pratique professionnelle selon le cadre imposé par le Code des professions : syndic et comité de discipline ${ }^{30}$, comité d'inspection professionnelle ${ }^{31}$, comité de révision ${ }^{32}$. La pratique de la sage-femme est régie par un code de déontologie ${ }^{33}$.

\section{ii. Contrôle de l'acte par l'établissement cocontractant}

La sage-femme qui conclut un contrat de services avec un CLSC fait partie des ressources humaines de l'établissement. La loi prévoit, à l'instar de la profession médicale, un pouvoir disciplinaire de l'établissement sur la pratique de la sage-femme. Le conseil d'administration peut prendre des mesures disciplinaires à l'égard d'une sage-femme. Ces mesures disciplinaires vont de la réprimande, de la modification ou de la privation de l'un ou plusieurs des droits prévus au contrat de services jusqu'à la résiliation de ce contrat. Toute mesure disciplinaire prise à l'endroit d'une sage-femme doit être motivée et fondée uniquement sur le défaut de qualification, l'incompétence, la négligence,

28. R.R.Q. 1981, c. S-0.1, r. 2 [Règlement sur l'accouchement à domicile].

29. L'application de ce règlement sera possible dès que l'assurance responsabilité des sages-femmes couvrira également le domicile : osfq, supra note 3.

30. Code des professions, supra note 7, art. 116.

31. Ibid., art. 90, 109.

32. Ibid., art. 123.3.

33. Ibid., art. 87, Loi sur les sages-femmes, supra note 1, art. 60. 
l'inconduite, l'inobservation des règlements de l'établissement ou le non-respect des obligations prévues à son contrat ${ }^{34}$.

La LSSSS prévoit la création d'un Conseil des sages-femmes pour chaque établissement public qui exploite un CLSC et qui a conclu, avec au moins cinq sages-femmes, un contrat de services ${ }^{35}$. Pour les autres cas, des alternatives sont possibles ${ }^{36}$. Sous réserve du pouvoir disciplinaire du conseil d'administration de l'établissement, le conseil des sages-femmes est responsable envers le conseil d'administration de contrôler et d'apprécier de manière générale la qualité et la pertinence des actes posés par les sages-femmes pour l'établissement ${ }^{37}$.

En outre, la LSSSS stipule qu'un responsable des services de sage-femme doit être nommé par tout établissement qui exploite un centre local de services communautaires où exercent des sages-femmes ${ }^{38}$. Cette personne doit surveiller et contrôler la qualité des actes posés par les sages-femmes pour l'établissement $^{39}$. Le responsable des services de sage-femme doit en outre élaborer des règles de soins que doit appliquer la sage-femme ${ }^{40}$. Il assume aussi des fonctions de coordination des services de sage-femme ${ }^{41}$. Dans l'exécution de ces fonctions, c'est un préposé de l'établissement.

Vu la nature du contrat qui lie une sage-femme et l'établissement, soit un contrat de services, ces mesures de contrôle et de surveillance n'auraient pas pour effet de créer un lien de préposition entre les parties.

34. LSSSS, supra note 17, art. 259.6.

35. Ibid., art. 225.1.

36. Ibid., art. 225.2, 225.3 in fine, 208.2.

37. Ibid., art. 225.3.

38. Ibid., art. 208.1.

39. Ibid., art. 208.2.

40. Ibid., art. 208.2 au para. 2.

41. Ibid., art. 208.3. 


\section{Responsabilité civile de la sage-femme}

La Loi sur les sages-femmes restreint le champ d'intervention professionnelle de la sage-femme à la grossesse normale. En effet, le Bureau de l'Ordre des sages-femmes doit déterminer les cas présentant un risque pour la femme ou son enfant, pendant la grossesse, le travail, l'accouchement et les six premières semaines de la période postnatale, et nécessitant en conséquence une consultation d'un médecin ou un transfert de la responsabilité clinique à un médecin, ainsi que les conditions dans lesquelles cette consultation ou ce transfert doit être effectué ${ }^{42}$. Le Règlement sur les cas nécessitant une consultation d'un médecin ou un transfert de la responsabilité clinique à un médecin ${ }^{43}$ détermine de façon assez précise ce qu'il faut entendre par «grossesse normale» en déterminant les cas de consultation médicale obligatoire et de transfert obligatoire de la responsabilité clinique à un médecin - ou si on préfère en identifiant les cas où la grossesse présente un risque. Ces dispositions s'appliquent en tous lieux : maison de naissance, centre hospitalier et domicile.

Le libre choix de la femme de consulter un médecin en tout temps n'est aucunement affecté par ces dispositions ${ }^{44}$. La sage-femme conserve aussi son autonomie professionnelle :

Si le bien de la cliente l'exige, la sage-femme doit consulter une autre sage-femme, un membre d'un autre ordre professionnel ou toute autre personne compétente, ou la diriger vers l'une de ces personnes. ${ }^{45}$

Comme le souligne l'auteure Caroline Simard, rien n'empêche la sage-femme d'initier une consultation médicale pour s'assurer que la grossesse de sa cliente est bel et bien une grossesse normale ${ }^{46}$.

42. $\quad$ Loi sur les sages-femmes, supra note 1, art. 5 (3).

43. R.R.Q. 1981, c. S-0.1, r. 1 [Règlement sur les consultations].

44. Code de déontologie des sages-femmes, art. 9, en ligne : Ordre des sages-femmes du Québec $<$ http://www.osfq.org/> [Code de déontologie]. La sage-femme doit reconnaître en tout temps le droit de la cliente de consulter une autre sage-femme, un membre d'un autre ordre professionnel ou une autre personne compétente.

45. Ibid., art. 14

46. Caroline Simard, supra note 4 à la p. 75. 
Lorsque la sage-femme identifie une condition médicale prévue aux annexes ${ }^{47}$ du Règlement sur les consultations, elle doit initier la consultation et informer la cliente des motifs de la consultation ${ }^{48}$. La sage-femme doit fournir au médecin consulté tous les renseignements et les documents pertinents à la consultation, en précisant le cas de consultation obligatoire ${ }^{49}$. À la suite de la consultation obligatoire, la sage-femme doit informer la femme des résultats de celle-ci ${ }^{50}$ et, en tenant compte des recommandations du médecin consulté ${ }^{51}$ :

[L]a sage-femme $[\ldots]$ :

$1^{\circ}$... poursuit son suivi;

$2^{\circ}$... poursuit son suivi pendant l'épisode de soins simultanés;

$3^{\circ}$... transfère la responsabilité clinique de la femme ou de l'enfant à un médecin. ${ }^{52}$

On voit donc une distinction fondamentale : un cas de consultation médicale obligatoire ne signifie pas automatiquement un transfert de la responsabilité clinique à un médecin.

La sage-femme doit transférer la responsabilité clinique de la femme ou de l'enfant à un médecin dans les cas de transfert obligatoire prévus au Règlement sur les consultations et informer la cliente des motifs du transfert ${ }^{53}$. Bien sûr, le transfert doit se faire dans des conditions optimales de sécurité pour la mère et l'enfant ${ }^{54}$ jusqu'à la prise en charge médicale en tenant en compte les circonstances (urgence, distance, conditions climatiques) où ce transfert a lieu ${ }^{55}$.

47. Le Règlement sur les consultations comporte plusieurs annexes selon les différentes étapes de la grossesse, du travail, de l'accouchement et du suivi postnatal de la mère et du nouveau-né.

48. $\quad$ Règlement sur les consultations, supra note 43, art. 1, 2.

49. Ibid., art. 3.

50. Ce qui inclut l'accès à son dossier. Code de déontologie, supra note 44, art. 32.

51. Évidemment, la recommandation du médecin doit être conforme au Règlement sur les consultations. On ne peut pas recommander un suivi par la sage-femme si la condition commande un transfert au médecin.

52. $\quad$ Règlement sur les consultations, supra note 43, art. 4.

53. Ibid., art. 5, 6 .

54. $\quad$ Code de déontologie, supra note 44, art. 17, 48 au para. 4, 54.

55. Règlement sur les consultations, supra note 43, art. 7-10. 


\section{a. Lien juridique sage-femme/parturiente}

Comme la sage-femme doit être rattachée à un CLSC par un contrat de services pour y pratiquer sa profession, la femme qui désire avoir recours aux services de sages-femmes doit s'y adresser. Il y a là de la part de la future maman une démarche volontaire de sollicitation de services professionnels.

La première question est celle de savoir s'il y a conclusion d'un contrat entre le CLSC et la cliente pour la fourniture de services de sages-femmes ou si un contrat de soins se crée entre la cliente et la sage-femme.

Cette distinction est très importante au niveau de la détermination du débiteur de soins : CLSC, sage-femme ou les deux. Nous croyons que le CLSC ne fait que mettre en contact la sage-femme et la cliente et ne fournit pas lui-même le service de sage-femme par autrui. La Cour d'appel dans la décision Camden-Bourgault c. Brochu ${ }^{56}$ a rejeté la théorie du contrat hospitalier selon laquelle l'hôpital serait le débiteur contractuel des soins de santé. Selon Me Jean-Pierre Ménard :

(La) principale conséquence du jugement de la Cour d'appel dans l'affaire Camden-Bourgault est de réduire considérablement la sphère de responsabilité hospitalière pour la faute médicale, en écartant toute possibilité de responsabilité de l'hôpital pour la prestation de soins médicaux. ${ }^{57}$

Quoique le CLSC permette l'accès aux services de sages-femmes, il ne paraît être qu'un facilitateur et non pas un fournisseur direct de soins ou de services. La nature de la relation contractuelle entre le CLSC et la sage-femme n'apparaît pas comme un facteur déterminant pour cette conclusion puisque la responsabilité contractuelle du CLSC pour le fait d'autrui n'en dépend pas. La question est plutôt de savoir si un contrat peut se former entre la cliente et le

56. [2001] R.R.A. 295 (C.A.).

57. Jean-Pierre Ménard, «La responsabilité hospitalière pour la faute médicale après la décision de la Cour d'appel dans l'affaire Camden-Bourgault : plus de questions que de réponses», dans Barreau du Québec, Service de la formation permanente, Le devoir de sécurité et la responsabilité des établissements de santé(2002), Cowansville (Qc), Yvon Blais, 2002, 139 à la p. 147. 
CLSC et si oui, si ce contrat inclut les services de sages-femmes. Pour les raisons invoquées plus haut, nous opinons qu'il ne se forme pas de contrat entre la cliente et le CLSC pour la fourniture des services de sages-femmes, ou qu'à tout le moins les obligations contractuelles du CLSC ne sont que des obligations matérielles d'organisation de la maison de naissance ou de structure des services de sages-femmes, mais sans inclure la prestation des services proprement dit.

Il ne faut pas croire que le CLSC soit à l'abri de tout recours. L'établissement a une obligation légale de prestation de soins sécuritaires ${ }^{58}$. Ainsi, il pourra être tenu personnellement responsable d'une déficience dans la fourniture de l'équipement requis ${ }^{59}$ ou d'une organisation inadéquate des ressources humaines ${ }^{60}$, telle une surcharge de travail ou une carence dans les services de garde ${ }^{61}$. Le CLSC répondra aussi de la faute de ses préposés, aides natales, infirmières, préposées aux bénéficiaires, responsable des services de sage-femme.

Comme on reconnaît la faculté de choix de la parturiente et le statut professionnel et autonome de la sage-femme, une conclusion s'impose. Un contrat de soins peut se former entre la cliente et la sage-femme. Le régime de la responsabilité civile contractuelle doit donc s'appliquer selon l'article 1458 C.c.Q. ${ }^{62}$. Cela implique de déterminer le contenu obligationnel du contrat et l'intensité de l'obligation assumée. Quant aux victimes par ricochet, seul le régime de responsabilité extracontractuelle trouvera application puisqu'aucun contrat ne se forme entre ces victimes et la sage-femme. On peut par ailleurs certainement imaginer des cas où une sage-femme fournit des soins à une parturiente sans qu'un contrat de soins ne se soit préalablement formé : le régime de responsabilité extracontractuelle prévaudra alors ${ }^{63}$. Mais, comme le précise Me Caroline Simard : «[q]uelle que soit la nature des rapports juridiques

58. $\quad$ LSSSS, supra note 17, art. 5, 80, 100, 101.

59. Weiss c. Solomon, [1989] R.J.Q. 731 (C.S.) (défaut d'avoir un réanimateur cardiaque disponible à l'étage).

60. Houde c. Côté, [1987] R.J.Q. 723 (C.A).. On peut ajouter des lieux inadéquats ou un système de communications déficient.

61. En droit français : Cour d'appel AMIENS, Chambre 1, 15 Octobre 2002, JurisData : 2002-196127.

62. C'est la summa divisio du droit de la responsabilité civile.

63. C.c.Q., art. 1457. 
qui unissent parturiente et sage-femme, celle-ci doit répondre de ses fautes si elle en commet» ${ }^{64}$.

\section{b. Devoirs et obligations}

La création d'une nouvelle profession avec un champ d'exercice exclusif nous oblige à en définir les devoirs et obligations. Établissons tout d'abord que la sage-femme ne saurait être assimilée à une infirmière ou à un médecin omnipraticien. Il s'agit d'une profession avec sa formation universitaire, ses exigences, ses contrôles, ses normes de pratique ${ }^{65}$ et son champ de pratique, la grossesse normale. Il ne s'agira pas de comparer la sage-femme avec des praticiens d'un autre ordre professionnel. Il serait erroné et contraire à l'intention du législateur de confondre la pratique de la sage-femme avec celle d'une infirmière bachelière, d'un médecin omnipraticien ou d'un résident de première année en obstétrique-gynécologie.

La pratique de la sage-femme se rapproche bien sûr de celle d'un médecin. Cependant, la pratique de la sage-femme se limite à la grossesse normale et comporte en plus des obligations impératives de consultation et de transfert dans des cas identifiés au Règlement sur les consultations. Le médecin n'a pas ces restrictions. Il demeure que le médecin a l'obligation déontologique, dans le cas de grossesse à risque élevé ou qui présente des risques particuliers, de consulter ou de transférer les cas qui dépassent ses compétences ${ }^{66}$. Sa pratique obstétricale dépendra aussi de ses privilèges hospitaliers et de la couverture d'assurance responsabilité qu'il a choisie. Mais, nous ne croyons pas que l'évaluation de la conduite professionnelle de la sage-femme doive se faire suivant le modèle du «bon médecin généraliste oeuvrant en obstétrique, placé dans les mêmes circonstances de temps et de lieu» ${ }^{67}$.

64. Caroline Simard, supra note 4 à la p. 75.

65. Code de déontologie, supra note 44, art. $3:$ :[1] sage-femme doit respecter les normes et principes énumérés par l'ICM (International Confederation of Midwives)». Voir en ligne : ICM <www.internationalmidwives.org >.

66. Code de déontologie des médecins, R.R.Q. 1981, c. M-9, r. 4.1, art. 42.

67. Contra Caroline Simard, supra note 4 à la p. 79. 
Cela dit, il n'est pas question pour nous de prétendre qu'il y a deux standards de soins selon le choix de la parturiente : le standard médical et le standard sage-femme. Ce serait intolérable. Une grossesse normale doit répondre à un standard de soins appropriés. L'expression de Me Caroline Simard, «règles de l'art obstétrical», nous apparaît particulièrement heureuse ${ }^{68}$. Dans tous les cas, dit la loi, les soins de santé doivent être adéquats sur les plans à la fois scientifique, humain, social, avec continuité et de façon personnalisée et sécuritaire $^{69}$.

Tout ce qui précède est applicable à la grossesse qui se déroule normalement, champ de pratique de la sage-femme. Dans l'hypothèse où la sage-femme fait défaut d'identifier un risque obstétrical qui fait dévier la grossesse de la normalité, omet de demander une consultation médicale ou de transférer, il ne peut plus être question du même modèle d'analyse. Les gestes posés par une sage-femme qui assume seule la responsabilité clinique d'une grossesse à risque doivent être comparés à celui du médecin omnipraticien ou spécialiste selon la condition. Un tel suivi est une faute civile : la sage-femme a fait ce que la loi lui interdit de faire. Mais il n'y aura responsabilité, si un dommage survient, que dans la mesure où on pourra dire qu'une intervention médicale aurait évité le dommage : la sage-femme, pas plus qu'un médecin, ne répond des aléas de la vie.

Les obligations professionnelles du médecin, développées par les auteurs Bernardot et Kouri ${ }^{70}$, nous apparaissent tout à fait appropriées à l'analyse de la responsabilité civile de la sage-femme. Nous examinerons plus particulièrement les obligations de renseigner, de soigner et de suivre.

Afin de donner un cadre pratique à ces obligations, nous nous inspirerons des recommandations du Conseil d'évaluation des technologies de

$68 . \quad$ Ibid.

69. LSSSS, supra note 17, art. 5.

70. Alain Bernardot et Robert P. Kouri, La responsabilité civile médicale, Sherbrooke, Éditions R.D.U.S., 1980. 
la santé ${ }^{71}$ et de la seule poursuite, à notre connaissance, intentée contre des sages-femmes québécoises ${ }^{72}$.

\section{i. L'obligation de bien renseigner}

L'obligation de la sage-femme de renseigner la parturiente est le corollaire nécessaire du principe de l'inviolabilité de la personne et de l'obligation d'obtenir un consentement libre et éclairé de la personne qui se soumet à des soins ${ }^{73}$.

Dans le cas d'une grossesse, il nous semble raisonnable de dire sans avoir peur de nous tromper que le désir normal des parents est d'accueillir l'enfant dans les meilleures conditions possibles même si le risque zéro n'existe pas et qu'on ne peut prévoir l'imprévisible ou déceler l'indécelable ${ }^{74}$. Le choix de la mère quant à la poursuite de sa grossesse lui appartient ${ }^{75}$.

Comme principes de base, mentionnons que la sage-femme doit chercher à établir une relation de confiance avec sa cliente et exercer sa profession de façon personnalisée ${ }^{76}$. La sage-femme doit être honnête envers la cliente ${ }^{77}$ et elle doit éviter de faire toute fausse représentation quant à son niveau de compétence ou quant à l'efficacité de ses services et de ceux généralement assurés par les sages-femmes ${ }^{78}$. En plus des avis et des conseils, la sage-femme doit fournir à sa cliente toutes les explications nécessaires à la compréhension et à l'appréciation des services qu'elle lui rend. Lorsqu'un choix de soins par la cliente est possible, la sage-femme doit lui fournir l'information lui permettant de faire un choix éclairée ${ }^{79}$.

71. CETS, Les mortinaissances, supra note 6.

72. Lachance et al. c. Beauchemin et al. [Règlement hors cour], Québec 200-05-008421-971, (C.S.) [affaire Mimosa] Voir aussi Richard Hénault, «Poursuite de 1,3M \$ contre 3 sages-femmes» Le Soleil (29 novembre 1997) A-4. En Ontario : Carere v. Cressman, [2002] O.T.C. 258 (C.S.).

73. C.c.Q., art. 10, 11.

74. Claveau c. Guimond, [1998] R.R.A. 616 (rés.).

75. Arndt c. Smith, [1997] 2 R.C.S. 539.

76. Code de déontologie, supra note 44, art. 11.

77. Ibid., art. 12.

78. Ibid., art. 13.

79. Ibid., art. 15. 
Le jugement rendu dans Labrie c. Gagnon illustre notre propos. Dans cette affaire, il s'agissait d'un cas d'accouchement vaginal après césarienne [AVAC] à l'occasion duquel les parents reprochaient à leur médecin de ne pas les avoir informés suffisamment des risques de rupture utérine. Devait-il le faire? La Cour d'appel conclut ainsi :

Il va sans dire, les trois experts reconnaissent que le choix appartient à la patiente et que, pour lui permettre d'exercer un choix éclairé, le médecin a l'obligation de lui fournir tous les renseignements requis incluant les risques inhérents à l'une ou l'autre méthode et, plus particulièrement, dans le cas de l'AVAC, le risque de rupture utérine. ${ }^{80}$

Selon le Règlement sur les consultations, l'AVAC n'apparaît pas être une contre-indication au suivi par une sage-femme. Toutefois, pendant le travail et l'accouchement, une suspicion de rupture utérine est un cas de transfert obligatoire et une césarienne doit être pratiquée rapidement, sinon d'urgence. Si la césarienne n'est pas pratiquée dans un très court délai, les risques de mortalité de l'enfant sont de $50 \%^{81}$. C'est un risque important qui doit être divulgué à la mère.

La cliente doit bien comprendre le champ d'intervention de la sage-femme, soit la grossesse normale. La sage-femme, par ailleurs, est dans la situation délicate où c'est elle qui doit reconnaître que la condition de la cliente présente un risque identifié au Règlement sur les consultations et, à notre avis, toute autre situation qui nécessiterait une consultation ou un transfert de responsabilité clinique et en informer la cliente ${ }^{82}$. Nous soumettons que la sage-femme doit même avoir une approche directive lorsque la consultation médicale est obligatoire. Elle ne peut minimiser une condition que le Règlement sur les consultations identifie comme nécessitant une consultation obligatoire ${ }^{83}$.

80. Labrie c. Gagnon, [2003] R.R.A. 400 (C.A.) au para. 19.

81. Ibid., au para. 19.

82. $\quad$ Règlement sur les consultations, supra note 43, art. 2.

83. La Loi sur les sages-femmes (art. 5, 6) et les règlements utilisent des expressions comme risque, condition anormale, gravité de la condition de la femme ou de l'enfant, préjudice qui pourrait en résulter, mesure d'urgence. 
La sage-femme n'est pas dans cette situation un simple diffuseur d'informations. Dans un tel cas, on peut douter que la sage-femme puisse légalement continuer un suivi alors que la consultation médicale obligatoire n'a pas eu lieu. Le Règlement sur les consultations fait peser sur la sage-femme l'obligation de s'assurer que la consultation soit tenue. N'y a-t-il pas obligation légale pour la sage-femme de s'abstenir d'agir si la consultation médicale obligatoire n'a pas lieu ${ }^{84}$ ? Si oui, et c'est notre opinion, un risque que la consultation médicale aurait pu éviter ne constitue plus un événement imprévisible et irrésistible ${ }^{85}$.

Selon le lieu de naissance, la sage-femme doit s'assurer que la cliente, particulièrement la primipare, comprenne bien les choix disponibles pour le contrôle de la douleur ${ }^{86}$. De plus, la parturiente doit être informée du recours ou non aux différentes techniques de monitoring foetal ${ }^{87}$. Le défaut d'une sage-femme de diagnostiquer un état de souffrance foetale ${ }^{88}$, faute de monitoring foetal adéquat, retardant d'autant la consultation médicale ou le transfert à un centre hospitalier, pourrait entraîner sa responsabilité civile ${ }^{89}$.

Dans le cas d'une grossesse à risque avec consultation médicale obligatoire, la sage-femme devra informer la cliente des résultats de celle-ci ${ }^{90}$. La possibilité d'un suivi de cette grossesse par la sage-femme demeure possible, sujet au choix éclairé de la cliente et aux recommandations médicales. Plus particulièrement, le refus d'une cliente de suivre une recommandation du

84. $\quad$ Code de déontologie, supra note 44, art. 14.

85. C.c.Q., art. 1470 .

86. À titre d'exemple, Roy c. Hôpital Ste-Justine, [1998] R.R.A. 241 (C.Q.).

87. Pratique médicale très usitée en milieu hospitalier. Voir Louise Hardy, Monitoring foetal et responsabilité de l'infirmière, essai de maîtrise soumis à la Faculté de droit, Université de Sherbrooke, 2002.

88. Cas de transfert obligatoire, Règlement sur les consultations, supra note 43.

89. En droit français : Cour d'appel TOULOUSE, Chambre 1 section 1, 13 Octobre 2003, JurisData : 2003-238433. Dans les autres provinces canadiennes, les cas de souffrance foetale donnent lieu à une abondante jurisprudence : Fullerton (Guardian ad litem of) v. Delair, 2005 B.C.S.C. 204; Allen (Next friend of) v. University Hospitals Board, 2000 A.B.Q.B. 509, conf. par 2002 A.B.C.A. 195; Bauer (Litigation guardian of) v. Seager, 2000 M.B.Q.B. 113; Granger (Litigation guardian of) v. Ottawa General Hospital, [1996] O.J. $\mathrm{n}^{\circ} 2129$ (Ont. Gen. Div.) (QL); McGlone (Guardian ad litem of) v. Kelly, 2002 B.C.S.C. 774; Guerineau (Guardian ad litem of) v. Seger, 2001 B.C.S.C. 291.

90. Règlement sur les consultations, supra note 43, art. 4. 
médecin consultant doit être bien documenté au dossier, notamment quant à ses implications pour la mère et l'enfant.

L'obligation de renseigner est une obligation continue tout au long de la prestation de soins. Le Règlement sur les consultations identifie d'ailleurs des situations de risque à tout moment de la grossesse, du travail, de l'accouchement et de la période postnatale.

Dans les affaires Reibl c. Hughes ${ }^{91}$ et Hopp c. Lepp ${ }^{92}$, la Cour suprême du Canada a établi que les risques importants devaient être divulgués au patient de façon à s'assurer de son consentement libre et éclairé. Un risque important est celui que la science identifie comme pouvant présenter des conséquences graves, quoique rares, ou fréquentes, quoique bénignes. De plus, doit être divulguée toute information qu'une personne raisonnable, placée dans les mêmes circonstances que le patient en l'espèce, jugerait important de savoir. On doit se placer du point de vue de la personne qui veut savoir et qui décide, le patient, et non du seul point de vue du débiteur de soins. Ainsi, dans l'affaire Reibl, le fait qu' une intervention chirurgicale importante aurait pu être reportée quelque temps sans préjudice imminent pour le patient, ce qui lui aurait permis de s'assurer du droit à une pension de retraite, a été jugé, en l'espèce, une information importante qui devait être divulguée. Le professionnel de la santé ne doit pas seulement énumérer les risques connus d'une procédure, mais également prendre le temps de connaître son patient et de dialoguer avec lui.

La patiente a également le droit de retirer le consentement donné et la sage-femme doit dans ce cas interrompre la procédure, sauf dans les cas où l'interruption de la procédure compromettrait la vie de la patiente ou de son enfant ou présenterait des problèmes graves et immédiats pour leur santé. Pour exercer ce droit de choisir, la Cour suprême du Canada ${ }^{93}$ a imposé au professionnel de la santé l'obligation de renseigner la patiente de tout changement important survenant au cours du traitement et de nature à influencer

91. [1980] 2 R.C.S. $880[$ Reibl].

92. [1980] 2 R.C.S. 192.

93. Ciarlariello c. Schacter, [1993] 2 R.C.S. 119. 
sa décision. Cela comprend certainement un changement important quant aux risques que comporte la procédure ou quant à la nécessité de la poursuivre.

La parturiente doit être informée de l'endroit où l'accouchement, sous les soins de la sage-femme, pourra avoir lieu. Comme on l'a vu, l'accouchement en milieu hospitalier n'est possible que si une entente entre le CLSC et un centre hospitalier a été conclue. L'accouchement à domicile présente des particularités au niveau de l'obligation de renseigner. En effet, un formulaire de consentement est prévu et la sage-femme doit fournir à la cliente les renseignements mentionnés sur ce formulaire afin de lui permettre de faire un choix éclairé. Le contenu de ce formulaire est très précis comme on peut le constater :

Je reconnais avoir été informée des éléments suivants :

- $\quad$ des particularités des différents lieux de naissance, des avantages et des risques qui y sont afférents;

- $\quad$ des mesures liées à l'accouchement à domicile;

- $\quad$ des cas où la consultation d'un médecin ou le transfert de la responsabilité clinique à un médecin est nécessaire;

- $\quad$ des mesures d'urgence à prendre lors d'une complication;

- $\quad$ des critères de transport du domicile au centre hospitalier lorsque indiqué, incluant l'implication de la distance. ${ }^{94}$

À notre avis, ce formulaire est indicatif du contenu de l'obligation de renseigner de la sage-femme même si l'accouchement n'a pas lieu au domicile, notamment quant aux «particularités des différents lieux de naissance, des avantages et des risques qui y sont afférents», aux cas de transfert de responsabilité clinique ou de consultation, de suivi conjoint, de transport d'urgence au centre hospitalier, etc. Si le risque zéro n'existe pas, manifestement le niveau de risque n'est pas le même selon le lieu de l'accouchement. Nécessairement, en cas de transfert, il y a aura des délais même avec une équipe très bien entraînée.

Le choix du lieu de naissance n'est pas un choix de dernière minute. Au moment où la femme fait ce choix, il n'y a pas d'urgence. Le choix lui-même

94. Règlement sur l'accouchement à domicile, supra note 28, ann. I. 
n'est pas urgent ${ }^{95}$. En soi, le choix du lieu de l'accouchement est purement électif si la grossesse est normale. La femme a tout le temps et le loisir de faire un choix éclairé, d'où l'importance d'une information complète. Jusqu'où la sage-femme doit-elle aller dans la divulgation des risques? Faut-il craindre qu'une divulgation trop complète ou trop franche effraie la femme et l'amène à renoncer à son désir d'accoucher naturellement à la maison? Penser de cette façon est justement le genre d'appropriation du pouvoir de décider, longtemps reproché aux médecins, en plus de nier complètement à la femme le droit et la capacité de choisir ${ }^{96}$. N'est pas meilleur le comportement de la sage-femme qui minimise les risques au point de donner l'impression qu' un accouchement hors hôpital ne présente aucun aléa. Une demi-vérité est-elle plus acceptable qu'un demi-mensonge?

En matière de responsabilité civile, l'expérience a démontré qu'un dossier bien tenu quant aux informations divulguées à la patiente et quant aux choix de celle-ci étaient d'un secours appréciable en cas de poursuite fondée sur un défaut de renseigner ${ }^{97}$. Devant des allégations précises d'une cliente qui connaît bien son cas, des allégations générales de bonne pratique habituelle, plusieurs années après le fait, font difficilement le poids ${ }^{98}$.

\section{ii. L'obligation de bien soigner}

La loi définit le champ de pratique de la sage-femme comme englobant tout acte ayant pour objet, lorsque tout se déroule normalement, de donner à une femme les soins et les services professionnels requis pendant la grossesse, le travail et l'accouchement et de donner à une femme et à son enfant les soins et les services professionnels requis durant les six premières semaines de la période postnatale. L'obligation de soigner de la sage-femme doit s'analyser à travers ce large éventail de soins.

95. Le Règlement sur l'accouchement à domicile prévoit l'éventualité d'un choix à la 36e semaine.

96. Dulude c. Gaudette, [1974] C.S. 618 aux pp. 621-622.

97. Ban c. Centre universitaire de santé McGill (CUSM) - Hôpital Royal Victoria, [2005] J.Q. no 3371 (C.S.) (QL).

98. Voir Labrie c. Gagnon, supra note 80 
Une partie importante du travail professionnel de la sage-femme, compte tenu de son champ de pratique limité, consistera justement à surveiller et à évaluer la condition de la parturiente aux divers stades de la grossesse, du travail, de l'accouchement et de la période postnatale par le dépistage de conditions anormales chez la femme ou son enfant ${ }^{99}$.

Qui dit dépistage, dit processus consciencieux de collecte, d'analyse et de contrôle des données pertinentes, le tout conformément aux bons usages scientifiques et professionnels. Le Règlement sur les consultations nous indique bien ce travail de recherche de la sage-femme au niveau des antécédents obstétricaux de la cliente puisque déjà, à ce stade, une grossesse présentant une condition à risque peut nécessiter une consultation médicale obligatoire ou un transfert de responsabilité clinique.

Tout au long du suivi de la grossesse, la sage-femme doit faire les tests et analyses requis par la bonne pratique, colliger l'information au dossier de la patiente, ajuster son suivi selon les circonstances et la condition médicale de la femme et de son foetus, et faire les recommandations appropriées à la parturiente afin de lui permettre de faire un choix éclairé ${ }^{100}$. Dans l'affaire Stéfanik c. Hôpital Hôtel-Dieu de Lévis ${ }^{101}$, un cas de grossesse multiple ${ }^{102}$, un médecin a été blâmé, à la suite d'une échographie, pour ne pas avoir procédé sans retard à d'autres examens plus approfondis devant des indices pouvant laisser soupçonner un retard de croissance intra-utérine ${ }^{103}$.

Il s'agit également des conclusions du Conseil d'évaluation des technologies de la santé dans son rapport ${ }^{104}$ :

99. Loi sur les sages-femmes, supra note 1, art. 6.

100. Carere v. Cressman, supra note 72 : défaut d'une sage-femme d'obtenir un examen par ultrason qui lui aurait permis de découvrir un placenta praevia et d'en informer la cliente; sage-femme tenue responsable de la paralysie cérébrale consécutive de l'enfant (indemnité de $2,3 \mathrm{M} \$$ ). À noter qu'il s'agit d'un cas où l'acte reproché a été posé en 1985, époque où la profession de sage-femme n'était pas réglementée en Ontario.

101. Stéfanik c. Hôpital Hôtel-Dieu de Lévis, [1997] R.J.Q. 1332 (C.S.).

102. Ce qui aurait été un cas de transfert obligatoire de la sage-femme à un médecin, Règlement sur les consultations, supra note 43, ann. II.

103. Le retard de croissance intra-utérine est également un cas de transfert obligatoire de la sage-femme à un médecin, ibid.

104. $\quad$ CETS, Les mortinaissances, supra note 6. 
Certains cas de mortinaissance analysés ont mis en évidence une difficulté à reconnaître la gravité d'un problème et à assurer un suivi adéquat pendant la période prénatale ou lors du travail.

Si le Règlement sur les consultations permet le suivi d'une grossesse par une sage-femme dans le cas de certaines conditions à risque identifiées, ce ne sera qu'à la suite d'une consultation médicale et du choix éclairé de la patiente. Me Caroline Simard énonce la règle de droit :

Toutefois, la sage-femme qui ne décèle pas une condition potentiellement dangereuse pour la mère ou le foetus parce qu'elle n'a pas procédé à une évaluation clinique consciencieuse (histoire de cas, examen physique, tests de laboratoire) commet une faute susceptible d'engager sa responsabilité. L'erreur est alors la résultante d'un processus fautif. ${ }^{105}$

Un autre élément doit être abordé. C'est la question de la «philosophie sage-femme, propre à sa profession» ${ }^{106}$. Le Code de déontologie précise :

La sage-femme doit respecter la philosophie du regroupement Les Sages-Femmes du Québec ${ }^{107}$ et ses modifications subséquentes ${ }^{108}$.

Avoir recours aux services de sages-femmes signifie-t-il une nécessaire adhésion aveugle et sans réserve de la parturiente à une philosophie, particulièrement celle d'un tiers? Y a-t-il danger de dérapage, voire de conflit, entre la philosophie de la sage-femme et les désirs de la parturiente ou encore avec les approches plus «médicales» de la maternité adoptées par d'autres professionnels de la santé? La parturiente n'est pas liée par le Code de déontologie des sages-femmes non plus que les autres professionnels de la

105. Caroline Simard, supra note 4 à la p. 83.

106. On retrouve sur le site web de l'Ordre des sages-femmes un énoncé de philosophie. Voir annexe ci-après.

107. Le Regroupement Les sages-femmes du Québec est l'association professionnelle des sages-femmes, voir en ligne : Regroupement les sages-femmes du Québec $<$ http://www.rsfq.org >.

108. Code de déontologie, supra note 44, art. 2. 
santé $^{109}$. Même si on reconnaît que la décision finale est celle de la femme, cette dernière sera nécessairement sensible aux recommandations de sa sage-femme, à qui elle fait confiance ${ }^{110}$. L'auteur Caroline Simard rapporte :

L'obligation de soigner comporte celle de référer en cas de besoin. À ce sujet, l'équipe d'évaluation des projets-pilotes a fait état de l'hésitation des sages-femmes à transférer les femmes vers les obstétriciens, en raison principalement de conflits interprofessionnels. Nous soumettons que les décisions de consultations [...] et de transferts doivent être dictées uniquement par la condition et les besoins médicaux de la mère et du nouveau-né. Procéder autrement constitue une faute. ${ }^{111}$

[...] L'équipe d'évaluation des projets-pilotes mentionne toutefois la tendance de certaines sages-femmes qui, motivées par une philosophie de non-intervention, repoussent les transferts au-delà des limites prescrites. ${ }^{112}$

Le retard à identifier une condition anormale et à transférer la patiente peut être la cause des séquelles subies par l'enfant. Non seulement la sage-femme doit être à l'écoute de sa cliente et porter une attention particulière à ses plaintes, elle doit réagir aux signaux d'alerte et se donner les moyens de les détecter ${ }^{113}$. Dans l'affaire Gravel c. Hôtel-Dieu d'Amos, les infirmières d'un centre hospitalier ont été jugées fautives d'avoir tardé à appeler le médecin malgré les instructions de ce dernier et les demandes répétées de la parturiente $^{114}$.

La philosophie de la sage-femme prône un «recours judicieux à la technologie». Qu'avec élégance ces choses sont dites, mais il ne faut pas croire

109. Par exemple, le médecin doit pratiquer la médecine selon des principes scientifiques, Code de déontologie des médecins, supra note 66, art. 6.

110. Labrie c. Gagnon, supra note 80 au para. 31 : «[i]l est tout aussi évident, comme le souligne le premier juge, que la recommandation du médecin que consulte le patient - comme tout autre professionnel d'ailleurs - est un élément très important dans l'exercice de son choix».

111. Caroline Simard, supra note 4 à la p. 85 [notes et parenthèses omises].

112. Ibid. aux pp. 86-87 [notes omises].

113. Cour d'appel TOULOUSE Chambre 1 section 1, 13 Octobre 2003, JurisData : 2003-238433.

114. Gravel c. Hôtel-Dieu d'Amos, [1989] R.J.Q. 64 (C.A.). 
que la preuve d'une pratique professionnelle largement répandue ou adoptée par les membres d'un ordre professionnel met à l'abri de l'examen judiciaire et de la responsabilité civile. Il faut que le caractère raisonnable de cette pratique puisse être démontré. La sage-femme qui adhère à une pratique professionnelle courante - ou une philosophie - qui n'est pas conforme aux normes générales de la responsabilité, c'est-à-dire que la conduite professionnelle doit être raisonnable et diligente, peut engager sa responsabilité selon les faits de l'affaire $^{115}$. Il ne sera pas suffisant de dire que «tout le monde fait ça » ou que «c'est comme ça que ça se passe» ou que «c'est contraire à notre philosophie». Dans G. v. C. et de Coster, le tribunal n'a pas hésité à intervenir pour corriger un usage professionnel imprudent ${ }^{116}$. De plus,

Le simple bon sens commande que, lorsque des méthodes simples pour éviter un danger ont été conçues, sont connues et sont accessibles, celui qui ne les emploie pas et provoque ainsi un résultat fatal ne peut pas prétexter que d'autres suivent la même pratique ancienne, moins prudente; que, lorsque ces méthodes sont aisément compréhensibles pour une personne ordinaire, par qui en outre la nécessité de les employer ou non est facilement comprise, il appartient autant au tribunal ou au jury qu'à des experts de trancher les questions; et que l'existence d'une pratique qui les néglige, même si elle est générale, ne peut pas protéger le chirurgien défendeur. ${ }^{117}$

Le monitoring foetal est un bon exemple ${ }^{118}$. Il s'agit d'une pratique courante et simple qui permet aux praticiens de détecter en temps utile un cas

115. Roberge c. Bolduc, [1991] 1 R.C.S. 374.

116. G. v. C. et de Coster, [1960] B.R. 161.

117. Anderson c. Chasney, [1949] 4 D.L.R. 71 (C.A. Man.), conf. par [1950] 4 D.L.R. 223

(C.S.C.). Traduction tirée de ter Neuzen c. Korn, [1995] 3 R.C.S. 674 au para. 49.

118. Me Caroline Simard, supra note 4 à la p. 86, rapporte : «Dans le cadre de nos recherches, nous avons assisté à une séance d'informations dans une maison de naissance [...] selon la sage-femme conférencière, le fait qu'il y ait peu d'équipement médical dans les maisons de naissance (par exemple à la maison Mimosa, on ne dispose pas du moniteur de coeur foetal) constitue un avantage puisque «cela permet aux sages-femmes d'éviter d'avoir à challenger les risques» de sorte que les transferts s'effectueraient plus tôt». Mme Louise Hardy explique que le monitoring foetal est un outil de choix pour évaluer la condition foetale. Par contre, son utilisation amène un taux plus élevé d'interventions obstétricales tels le recours à la césarienne ou l'utilisation des forceps. Ibid. à la p. 6. 
de souffrance foetale ${ }^{119}$. On a déjà jugé qu'un médecin doit suivre les signaux du moniteur foetal et réagir adéquatement :

The issue is not whether Dr. Kelly could have made an honest error of judgment in interpreting the strip, or whether he could have come to the conclusion that the data which was produced by the fetal heart monitoring equipment did not suggest intervention, but the issue is that he was not paying attention and did not even notice that significant accelerations in the fetal heart beat were not associated with the contractions of labour.

The standard of care of application to Dr. Kelly required that he observe and consider the information available to him from the fetal heart monitoring.

[...] I am of the opinion that Dr. Kelly was negligent in not paying adequate attention to the data being produced by the fetal heart monitoring equipment and in not noticing that rapid accelerations in the rate of the fetal heart were not associated with uterine contractions, and that Dr. Kelly's negligence was a material cause of Taylor's birth not being expedited and her being injured at birth by asphyxiation. ${ }^{120}$

Le fait qu'une maison de naissance ne dispose pas d'un moniteur foetal ou que la sage-femme n'y ait pas recours est-il fautif? La sage-femme n'a pas d'obligation légale de consulter un médecin si la grossesse est normale mais elle doit prescrire, effectuer et interpréter les examens et analyses de la bonne pratique obstétricale. Chose sûre, en cas d'accident, les décisions de la sage-femme seront scrutées attentivement. Dans certaines circonstances, la survenance d'un cas de transfert obligatoire ou d'un autre cas d'urgence pourra nécessiter le monitoring foetal de façon à informer le médecin du rythme cardiaque de l'enfant et à préparer l'intervention médicale. S'il n'y en a pas eu, comment anticiper une condition anormale et assurer un transfert sécuritaire?

119. En cas de souffrance fotale en cours de travail ou d'accouchement, la parturiente doit être transférée à un médecin, Règlement sur les consultations, supra note 43, ann. III au para. 11 .

120. McGlone (Guardian ad litem of) v. Kelly, supra note 89 aux para. 235, 236, 245. 
Dans l'affaire Carere v. Cressman, un tribunal ontarien avait à déterminer si le recours à un examen à l'ultrason était une pratique que devait suivre une sage-femme en 1985, même si cette dernière ne pouvait pas prescrire un tel examen à cette époque, la profession de sage-femme n'étant pas réglementée :

Given the fact that there may be an undetected placenta previa ${ }^{121}$, and given the risks of performing an ECV (pour «external cephalic version» - version céphalique externe ${ }^{122}$ ) in the presence of a placenta previa, is it reasonable to expect a midwife to direct the patient to obtain an ultrasound examination through her medical doctor to determine the location of the placenta before the midwife performed an ECV?

In my view, the answer to this question is found by examining the risks to the patient if the midwife performs the ECV without knowing the location of the placenta, and by examining the ease with which an ultrasound examination could be obtained.

[...] Therefore, I find that a reasonable midwife in 1985 in Ontario would be expected to direct the patient to obtain an ultrasound examination through her medical doctor, or the local hospital, to determine the location of the placenta before the midwife performed an ECV. I also find that this standard of care must be even more strictly followed if the midwife knew, or ought to have known, that the risk of complications that was greater than usual. ${ }^{123}$

Le fait pour la sage-femme de ne pas avoir recommandé l'ultrason comme moyen de diagnostic a également violé son obligation de renseigner la

121. Insertion anormale du placenta dans le segment inférieur de l'utérus : Marcel Garnier et Jacques Delamare, Dictionnaire illustré des termes de médecine, $28^{\mathrm{e}}$ éd., Paris, Maloine, 2002 [Garnier Delamare]. Au Québec, risque nécessitant un transfert obligatoire, Règlement sur les consultations, supra note 43, ann. III au para. 10.

122. Version : changement de position que l'on imprime au foetus pour faciliter sa sortie de l'utérus. Elle est dite céphalique quand on amène la tête du foetus vers le petit bassin et externe car on la pratique en imprimant au foetus des mouvements à travers la paroi : Garnier Delamare, supra note 121.

123. Carere v. Cressman, supra note 72 aux para. 92, 93, 97. 
parturiente madame Carere. Or, le tribunal estime que si la mère avait été bien informée, il est raisonnable de penser qu' elle aurait refusé la procédure proposée (version céphalique externe) :

However, a reasonable patient in Carere's particular position would still not act foolishly even if she was an ardent believer in home birth. If the serious consequences of performing an ECV in the presence of a placenta previa were explained, and if the patient was told that she was at a higher risk of having a placenta previa, I find that a reasonable patient in Carere's particular position would have probably declined to have the ECV.

Furthermore, if the same information had been fully explained to the patient, and if the patient was told that she could adequately determine the risk by having a simple ultrasound examination, I find that a reasonable patient in Carere's particular position would have elected to have the ultrasound examination before having the ECV. If so, the ultrasound would have detected the placenta previa and the ECV would not have been performed. ${ }^{124}$

\section{iii. L'obligation de suivre}

S'il est un volet sur lequel l'Ordre des sages-femmes insiste, c'est bien celui du suivi. Et c'est également sur cet aspect que les femmes ont le plus réclamé de services. Alors que le suivi médical traditionnel se limite souvent à une visite mensuelle, les autorités sanitaires ont réagi en mettant sur pied des cours prénatals et un suivi post partum par le CLSC. Il demeure que la parturiente a besoin de plus d'aide et de support malgré les efforts consentis par sa famille et son entourage qui sont des néophytes, particulièrement lors de l'accouchement et du travail.

Selon l'Ordre des sages-femmes :

Le suivi de la sage-femme va bien au-delà de la stricte naissance du bébé. La relation entre la sage-femme et la future maman commence dès le début de la grossesse et dure jusqu'à la période postnatale. La

124. Ibid., aux para. $120,121$. 
professionnelle est là tout au long des processus physiologiques normaux se présentant au cours de la grossesse ainsi que pendant et après l'accouchement. Elle guide la femme et sa famille à traverser cette grande étape de transformation. ${ }^{125}$

Afin d'assurer un suivi adéquat à la cliente, la pratique de la sage-femme est organisée en pratique de groupe ${ }^{126}$. La cliente choisit ou se fait assigner une sage-femme. Celle-ci est jumelée à une autre sage-femme, la coéquipière, que la cliente rencontrera pendant sa grossesse. Quoique l'on s'attende à ce que la sage-femme responsable soit présente à l'accouchement dans la majorité des cas, il peut arriver que ce soit la coéquipière qui l'accompagne lors de l'accouchement ${ }^{127}$. Cela signifie que la coéquipière devra connaître le dossier aussi exhaustivement que la sage-femme responsable. La coéquipière pourra également seconder la sage-femme responsable lors de la naissance de l'enfant ou la remplacera, au besoin ${ }^{128}$. La coéquipière assurant les soins d'une cliente pendant l'absence de la sage-femme responsable doit transmettre à celle-ci, à son retour, toute information utile à la continuité des soins pour cette cliente ${ }^{129}$.

Dans le cas du transfert obligatoire de la responsabilité clinique à un médecin, la sage-femme doit prendre les mesures appropriées pour faciliter le transfert en fonction de la nature du cas de transfert obligatoire ${ }^{130}$. La sage-femme qui se trouve avec la femme (sage-femme responsable ou coéquipière) ou l'enfant au moment du transfert doit l'accompagner jusqu'à la prise en charge médicale lorsque les circonstances le requièrent ${ }^{131}$. La sage-femme doit fournir au médecin tous les renseignements et les documents

125. Osfq, supra note 3 (à spécificité de la pratique).

126. C'est un mode de pratique que l'on rencontre chez les médecins obstétriciens. Voir par exemple, Drapeau-Gourd c. Power (16 mars 1982), Montréal 500-05-004294-755, J.E. 1982-424 (C.S.).

127. On comprend la nécessité d'une stricte tenue du dossier obstétrical.

128. Mimosa, supra note 23.

129. Code de déontologie, supra note 44, art. 55.

130. Règlement sur les consultations, supra note 43, art. 7.

131. Règlement sur les consultations, supra note 43, art. 8 . 
pertinents à la prise en charge de la parturiente ou de l'enfant, en précisant le cas de transfert visé au règlement ${ }^{132}$.

Que se passe-t-il si la femme refuse la consultation médicale ou le transfert obligatoires? La personne étant inviolable, une parturiente apte peut refuser un soin même bénéfique ${ }^{133}$. Un refus éclairé de soins est valable en droit. On ne peut pas non plus forcer la femme à recevoir un soin médical, même si la vie ou la santé de son foetus peut être en jeu ${ }^{134}$. Mais la sage-femme doit-elle continuer à suivre cette parturiente? Nous ne le croyons pas. Si la grossesse dévie de la normalité, la sage-femme doit se retirer. Toute convention visant à exclure ou limiter la responsabilité civile de la sage-femme serait sans effet ${ }^{135}$. Le désir, voire l'obstination de la parturiente, ne peut rendre légal ce qui est illégal. En droit, il s'agit d'un refus de collaborer aux soins et la sage-femme peut mettre fin au contrat ${ }^{136}$. En fait, l'exécution du contrat de soins est face à une impossibilité d'exécution juridique, la loi ne permettant pas à la sage-femme de continuer à suivre cette parturiente ${ }^{137}$. Le retrait de la sage-femme n'est donc pas susceptible de responsabilité civile.

La sage-femme ne pourra pas non plus être taxée d'avoir abandonné sa cliente. Il n'y a pas urgence et des soins de santé sont tout à fait disponibles par d'autres professionnels. Si la sage-femme passe outre à l'interdiction légale, la prestation de soins n'est plus sécuritaire au sens de la loi et devant ce cas de négligence, le CLSC serait bien fondé d'exercer son pouvoir disciplinaire et de résilier le contrat de services ${ }^{138}$. Une plainte déontologique, pour avoir

132. Règlement sur les consultations, supra note 43, art. 9; Code de déontologie, supra note 44, art. 54.

133. C.c.Q., art. 10, 11 C.c.Q. Robert P. Kouri et Suzanne Philips-Nootens, Le corps humain, l'inviolabilité de la personne et le consentement aux soins, Sherbrooke, Éditions R.D.U.S., 1999 aux para. 297, 336 et s. Pauline Lesage-Jarjoura et Suzanne Philips-Nootens, Éléments de responsabilité civile médicale : le droit dans le quotidien de la médecine, $2^{\mathrm{e}}$ éd., Cowansville (Qc), Yvon Blais, 2001 au para. 219 [Lesage-Jarjoura et Philips-Nootens].

134. Office des services à l'enfant et à la famille de Winnipeg (région du Nord-Ouest) c. G. (D.F.), [1997] 3 R.C.S. 925.

135. C.c.Q., art. 1474; Code de déontologie, supra note 44, art. 19.

136. Lesage-Jarjoura et Philips-Nootens, supra note 133 au para. 37.

137. C.c.Q., art. 1693.

138. LSSSS, supra note 17, art. 259.6. 
outrepassé ses compétences, pourrait être portée contre la sage-femme ${ }^{139}$. On peut même penser qu'un tel suivi serait un risque exclu par l'assureur.

Toujours dans cette même hypothèse, si l'accouchement tourne mal, la responsabilité civile de la sage-femme est-elle engagée? Supporte-t-elle tous les aléas de l'accouchement ou seulement les conséquences de ses gestes? Un tel suivi est certainement une faute civile. Il n'y a pas de présomption de causalité entre le suivi illégal et un préjudice à l'enfant. Par contre, si la sage-femme s'engage dans une activité risquée sans avoir les compétences que la loi prescrit, elle ne pourra plaider qu'elle a fait de son mieux. Dans cette hypothèse, les gestes de la sage-femme seront comparés à ceux d'un spécialiste des grossesses à risques ${ }^{140}$.

Pourrait-on plaider que la «faute de la mère» est la seule cause du dommage? Exercer son droit fondamental de refuser des soins n'est certainement pas une faute. Au mieux, il s'agirait d'une acceptation des risques mais pas d'une renonciation à exercer un recours en responsabilité civile ${ }^{141}$. La sage-femme serait-elle excusée? L'affirmer serait d'avaliser une pratique interdite par la loi.

Si le geste fautif de la sage-femme cause un préjudice à l'enfant et qu'il naît viable, un recours en responsabilité civile, par le représentant légal de l'enfant $^{142}$, est possible contre la sage-femme. La sage-femme pourrait-elle alors intenter un recours récursoire contre la mère pour avoir refusé la consultation médicale ou le transfert? Il faudrait alors conclure que la mère avait le devoir de se faire soigner, ce qui n'est pas possible selon la Cour suprême du Canada ${ }^{143}$. La responsabilité reviendrait alors entièrement à la sage-femme ${ }^{144}$.

\footnotetext{
139. Code de déontologie, supra note 44, art. 8, 17, 48(4).

140. Lesage-Jarjoura et S. Philips-Nootens, supra note 133 au para. 305.

141. C.c.Q., art. 1477.

142. Ibid., art. 159.

143. Office des services à l'enfant et à la famille de Winnipeg (région du Nord-Ouest) c. G. (D.F.), supra note 134.

144. C.c.Q., art. 1481.
} 
La sage-femme n'est pas non plus dans la situation d'un bon samaritain et ne pourra profiter de l'immunité partielle de l'article 1471 C.c.Q. La sage-femme ne porte pas secours à la parturiente en acceptant de continuer à la suivre. D'ailleurs, la Loi sur les sages-femmes envisage les cas d'urgence et lui permet de poser certains actes médicaux lorsque, justement, l'aide médicale n'est pas disponible à temps ${ }^{145}$. La sage-femme ne peut provoquer une situation d'urgence pour ensuite l'invoquer afin de s'exonérer.

\section{Pluralité des intervenants et responsabilité plurale}

La naissance doit être un événement heureux mais ce n'est pas toujours le cas. Selon le Rapport sur les soins de santé au Canada 2004 de l'Institut canadien d'information sur la santé, les traumatismes à la naissance surviennent chez près de 11,6 cas parmi 1000 naissances à l'hôpital, soit 1 traumatisme pour 81 nouveau-nés. Les soins périnatals étant souvent multidisciplinaires, il est nécessaire de se pencher sur la pluralité des intervenants et la responsabilité qui peut en découler.

La Loi de Murphy veut que les catastrophes aient une fâcheuse tendance à se produire. L'affaire Mimosa est certainement l'exemple-type du «worst case scenario». Résumé succinctement, il s'agit du suivi d'une grossesse à risque par des sages-femmes alors qu'il aurait dû y avoir un transfert de responsabilité clinique à un médecin ou, à tout le moins, une consultation médicale ${ }^{146}$. L'accouchement a eu lieu dans une maison de naissance et le nouveau-né s'est rapidement trouvé en détresse cardio-respiratoire. Branle-bas de combat général : l'enfant, très mal en point, doit être transféré d'urgence à l'hôpital. Les médecins reçoivent l'enfant dans un état catastrophique, sans avoir le dossier, et perdent du temps à chercher la pathologie. Les analyses biologiques n'ont pas été faites et le placenta a été détruit à la maison de naissance. C'est le cauchemar d'un médecin de garde.

145. Loi sur les sages-femmes, supra note 1, art. 6.

146. Rupture des membranes chez une parturiente, porteuse connue de streptocoque du groupe B. Selon nous, les gestes posés par une sage-femme qui assume seule la responsabilité clinique d'une grossesse à risque doivent être comparés à celui du médecin omnipraticien ou spécialiste selon la condition. 
La méthode d'analyse de la responsabilité de la sage-femme n'est pas différente de la méthode classique applicable en responsabilité civile. Après avoir déterminé les devoirs légaux ou contractuels de la sage-femme, il faut déterminer s'il y a eu faute, c'est-à-dire violation de ces devoirs. Vu la nature de la prestation de soins, où l'aléa est toujours possible, on peut qualifier généralement les obligations de la sage-femme comme étant des obligations de moyen $^{147}$, ce qui implique l'appréciation in abstracto de son comportement. Ainsi, le comportement de la sage-femme à un moment donné devra être comparé à celui d'une praticienne prudente et diligente, placée dans les mêmes circonstances de temps et de lieu, ce qui est une norme objective. Le fait que la profession de sage-femme soit récente et que les premières cohortes d'étudiantes universitaires seront bientôt diplômées fera en sorte que les normes de pratique de la sage-femme québécoise devront être déterminées par les tribunaux. À notre point de vue, la détermination des normes de pratique acceptables ne se fera pas dans l'abstrait ou seulement à partir de ce que les sages-femmes québécoises estiment être la «bonne pratique obstétricale». On aura recours aux ouvrages scientifiques reconnus et faisant autorité et aux pratiques des sages-femmes d'autres provinces ou formées à l'étranger. Les médecins de famille, obstétriciens et infirmières en périnatalité seront consultés, par exemple quant aux risques associés à telle ou telle complication ou condition ${ }^{148}$, aux signes avant-coureurs, aux mesures préventives et de surveillance. La détermination de la «bonne pratique obstétricale» n'appartient pas qu'à une seule catégorie de professionnels de la santé.

L'arrivée d'une nouvelle professionnelle en périnatalité fera en sorte de rendre l'analyse juridique plus complexe en matière de pluralité d'intervenants. Il n'est pas de notre intention de passer en revue la multitude de cas qui pourraient survenir, au risque d'en omettre, mais plutôt d'examiner les paramètres pertinents à cette analyse.

147. Ce n'est pas toujours le cas. Par exemple, en cas de transfert, la transmission en temps opportun des renseignements et des documents pertinents à la prise en charge médicale, nous apparaît être une obligation de résultat.

148. Voir la méthodologie du tribunal dans Carere v. Cressman, supra note 72 au para. 84 : «Because there was no recognized practice for midwives who performed ECVs [external cephalic version] in Ontario as of 1985, it is for the court to determine the reasonable standard of care expected of a midwife who attempted to do so». 


\section{a. Paramètres généraux}

Selon nous, un contrat de soins peut se créer entre une parturiente et une sage-femme, ayant pour objet les soins de santé. Ce contrat de soins comporte plusieurs obligations, généralement des obligations de moyens. La sage-femme doit cependant s' assurer que ce contrat peut légalement se former et être exécuté c'est-à-dire déterminer si, en tout temps pertinent, la grossesse évolue normalement. Tout écart dans le déroulement de la grossesse, le travail, l'accouchement ou la période postnatale peut obliger la sage-femme à transférer la patiente à un médecin. Cet écart n'est pas, à notre avis, limité à la nomenclature des risques énumérés au Règlement sur les consultations mais à toute condition qui, selon les règles de l'art obstétrical, nécessiterait une consultation médicale ou un transfert.

En présence de faute causale d'un préjudice et dans l'hypothèse précitée, la responsabilité de la sage-femme serait de nature contractuelle ${ }^{149}$.

149. C.c.Q., art. 1458. 


\section{b. Paramètres quant à la sage-femme}

La sage-femme répond de la faute qu'elle commet à titre de sage-femme responsable ou à titre de coéquipière. À notre avis, lorsque la sage-femme responsable est assistée d'une coéquipière, il ne s'agit pas de délégation de soins, entraînant une responsabilité contractuelle de la sage-femme responsable pour le fait d'autrui. La sage-femme responsable ne fait qu'exécuter ses propres obligations de diligence, en assurant le suivi de la parturiente ou en se faisant assister par une professionnelle qualifiée. Si c'est la coéquipière qui pratique seule l'accouchement et qu'elle commet une faute, seule la responsabilité de cette dernière pourra être recherchée, à moins que l'on puisse dire que le remplacement a été fautif, comme ce serait le cas si la coéquipière n'avait pas le degré d'expertise requis pour le $\operatorname{cas}^{150}$. Si chaque sage-femme commet une faute, on pourra conclure à une responsabilité in solidum ${ }^{151}$. Telle serait l'hypothèse où une sage-femme commet une faute (par exemple une faute de prescription de médicament), que la coéquipière aurait dû détecter.

\section{c. Paramètres quant au lieu de naissance}

La parturiente doit être informée de ses choix, s'il en est, en matière de lieux de naissance : maison de naissance, centre hospitalier ou domicile. La sage-femme doit, en outre, tenir compte des recommandations du médecin consulté et le cas échéant, les transmettre à la cliente de façon à lui permettre de faire un choix éclairé.

Même si l'accouchement a lieu en maison de naissance, la sage-femme peut, ou dans certains cas doit, requérir une consultation médicale ou un transfert. C'est l'obligation du CLSC de conclure avec un centre hospitalier une entente quant au soutien médical ${ }^{152}$. Le médecin consulté n'a pas le contrôle ni ne dirige la sage-femme lors de l'accouchement à la maison de naissance. Il répond toutefois de ses propres fautes comme tout professionnel. Le médecin devra être particulièrement prudent dans les cas de consultation médicale à

150. En matière médicale, voir Currie c. Blundell, [1992] R.J.Q. 764 (C.S.).

151. Ibid.

152. LSSSS, supra note 17, art. 259.11. 
distance, surtout lorsqu'il n'a pas accès aux tracés et autres données essentielles à son diagnostic. Cela n'exonère pas la sage-femme si elle tarde à appeler le médecin en consultation, à colliger les données nécessaires au diagnostic ou à initier le transfert.

L'accouchement en centre hospitalier par une sage-femme n'est possible que si une entente a été conclue entre le CLSC et le centre hospitalier. Comme professionnelle, la sage-femme ne devient pas une préposée momentanée du centre hospitalier. Ce dernier n'exerce aucun contrôle ou direction sur l'acte professionnel $^{153}$. Le centre hospitalier est toutefois tenu des obligations auxquelles il s'est engagé par le biais de l'entente conclue avec le CLSC, obligations au bénéfice des usagères et des sages-femmes ${ }^{154}$. Le centre hospitalier répond cependant des fautes de ses préposés, telles les infirmières de son service d'urgence ou d'obstétrique ${ }^{155}$.

Le fait qu' une collaboration doive être prévue entre la sage-femme et les ressources médicales du centre hospitalier n'entraîne pas une responsabilité des médecins pour les fautes de la sage-femme. La sage-femme n'est pas dans la situation où elle pose des actes médicaux par délégation sous une surveillance médicale immédiate. Le médecin de garde n'a pas à être présent de façon continue sauf si la situation l'exige. De plus, qu'un médecin traitant soit assisté par une sage-femme n'en fait pas non plus ipso facto le commettant de cette dernière, pas plus qu'il ne devient commettant des infirmières qui l'assistent. Le médecin répond toutefois de sa propre faute : ainsi, s'il laisse une patiente aux soins d'une sage-femme dépassée ou surchargée, il pourra être trouvé responsable ${ }^{156}$.

La jurisprudence québécoise a toutefois reconnu qu'un médecin pouvait voir sa responsabilité civile engagée pour les fautes des médecins résidents à qui il a délégué des tâches médicales, en l'espèce le suivi d'un patient qui dépassait

153. Camden-Bourgault c. Brochu, supra note 56.

154. LSSSS, supra note 17, art. 259.10.

155. Gravel c. Hôtel-Dieu d'Amos, supra note 114.

156. Cour d'appel PARIS Chambre 1 section B, 23 Septembre 2004, JurisData : 2004-254024. 
le cadre normal du suivi infirmier ${ }^{157}$. Ce serait aussi le cas du médecin qui, devant exécuter lui-même l'accouchement, se substitue la sage-femme. La réciproque est aussi vraie : une sage-femme qui se substitue une aide natale ${ }^{158}$ ou une sage-femme stagiaire pour l'exécution des actes qu'elle doit poser elle-même répond de la faute de cette dernière.

Dans une affaire française, on a jugé qu'un obstétricien avait commis une faute de surveillance pour avoir négligé de s'être rendu au chevet de la parturiente, bien qu'informé par la sage-femme, par téléphone, de la présence de liquide méconial lors de la rupture des membranes, indice évident de souffrance foetale. En l'espèce, le médecin ne pouvait valablement se retrancher derrière les indications rassurantes de la sage-femme, quant à l'apparente normalité des tracés de monitoring, laissés à la seule interprétation de cette dernière. Le praticien devait consulter lui-même le tracé de l'enregistrement du coeur foetal afin de vérifier directement le rythme des battements ${ }^{159}$.

En cas de pluralité de fautes causales distinctes, dont chacune aurait pu entraîner le dommage mais sans qu' on puisse déterminer avec certitude laquelle l'a effectivement causé, une responsabilité solidaire est prévue ${ }^{160}$. Ce sont les codébiteurs qui ont alors l'obligation de faire déterminer les quotes-parts de chacun dans la condamnation ${ }^{161}$.

Dans le cas d'un accouchement à domicile, des exigences matérielles sont requises et doivent être vérifiées par la sage-femme avant l'accouchement ${ }^{162}$. La sage-femme doit de plus avoir en sa possession lors de

157. Labrecque c. Hôpital du St-Sacrement, [1995] R.R.A. 510 (C.S.), conf. par [1997] R.J.Q. 69 (C.A.). Aussi Goupil c. Centre hospitalier universitaire de Québec (Pavillon Hôtel-Dieu de Québec), [2001] R.J.Q. 1814 (C.S.) [Goupil]; Murray-Vaillancourt c. Clairoux, [1989] R.R.A. 762 (C.S.).

158. Il s'agit d'une préposée aux bénéficiaires qui apporte un soutien aux sages-femmes d'une maison de naissance. La délégation d'actes à cette aide natale doit être forcément restreinte à des actes purement accessoires. Voir Mimosa, supra note 23.

159. Cour d'appel PAU Chambre 1, 21 Décembre 2000, JurisData : 2000-138044.

160. C.c.Q., art. 1480; Leroux c. Hôtel-Dieu d'Arthabaska, [1990] R.R.A. 378 (C.A.).

161. C.c.Q., art. 1478, 1529, 1537. C.p.c., art. 469.

162. Règlement sur l'accouchement à domicile, supra note 28, art. 4, 5 . 
l'accouchement, le dossier de la parturiente et tous les équipements énumérés au Règlement sur l'accouchement à domicile.

En cas de transfert d'urgence, la sage-femme doit s'assurer qu'il se déroule en toute sécurité pour la mère et son bébé jusqu'à la prise en charge médicale ${ }^{163}$.

\section{d. Paramètres quant aux risques obstétricaux}

La sage-femme doit initier une consultation d'un médecin dans les cas de consultations obligatoires prévus par l'une des annexes du Règlement sur les consultations ainsi que dans tous les cas où le bien de la parturiente l'exige ${ }^{164}$. Elle doit s'assurer que la consultation soit tenue dans un délai raisonnable, compte tenu de la gravité de la condition de la femme ou de l'enfant et du préjudice qui pourrait en découler ${ }^{165}$. À la suite de cette consultation, la sage-femme doit informer la femme des résultats de celle-ci et, en tenant compte des recommandations médicales ${ }^{166}$.

\section{i. La sage-femme poursuit le suivi de la grossesse}

Dans cette hypothèse, le médecin consulté ne répond que de la conformité scientifique et réglementaire de sa recommandation. La sage-femme répond, seule, de sa responsabilité professionnelle. Peut-elle faire fi de la recommandation médicale? Il faudrait d'abord que la patiente soit dûment informée et fasse un choix éclairé et libre quant au suivi en solo de la sage-femme. En cas de désaccord entre la sage-femme et le médecin, la voie la plus sûre serait certainement de demander une consultation auprès d'autre médecin.

163. Code de déontologie, supra note 44, art. 17, 48(4) (54). Règlement sur les consultations, supra note 43, art. 5-10, Règlement sur l'accouchement à domicile, supra note 28, art. 8; généralement sur les obligations en cas de transfert, Lapointe c. Hôpital Le Gardeur, [1989] R.J.Q. 2619 (C.A.).

164. Code de déontologie, supra note 44, art. 14.

165. Règlement sur les consultations, supra note 43, art. 1.

166. Ibid., art. 4. 
Si la sage-femme écarte sans raison la recommandation médicale et que se réalise un risque que la recommandation avait justement pour but d'éviter, elle pourra en répondre ${ }^{167}$. La parturiente n'est pas une cliente captive et seul son bien doit guider la sage-femme ${ }^{168}$.

\section{ii. Il y a suivi conjoint sage-femme/médecin}

Dans cette hypothèse, chaque professionnel répond de sa faute. La faute de la sage-femme n'entraîne pas la responsabilité du médecin à moins que l'on puisse dire qu'il y a eu faute contributive du médecin.

L'hypothèse d'un novus actus interveniens demeure possible dans le cas où la faute de la sage-femme est suivie d'une faute médicale qui, si elle n'avait pas été commise, aurait permis d'éviter la survenance du dommage ${ }^{169}$. Seul le médecin serait alors responsable. Mais il faut conclure à une véritable rupture du lien causal et ne pas confondre cette hypothèse avec celle des fautes successives mais contributoires qui entraîne une condamnation solidaire ${ }^{170}$.

\section{iii. Il y a transfert de la responsabilité clinique}

Le Règlement sur les consultations prévoit le transfert dans certains cas identifiés. Il y aura également transfert lorsque le bien de la parturiente l'exige ${ }^{171}$ ou s'il y a urgence, soit lorsque le cas dépasse les compétences de la sage-femme et que la vie ou la santé de la mère et de son enfant sont en péril. Par ailleurs, la sage-femme est autorisée à poser certains actes en cas d'urgence :

Constitue également l'exercice de la profession de sage-femme, en cas d'urgence et dans l'attente d'une intervention médicale requise ou en l'absence de celle-ci, le fait d'appliquer la ventouse, de pratiquer l'accouchement en présentation du siège, de pratiquer l'extraction

167. McCormick c. Marcotte, [1972] R.C.S. 18.

168. Code de déontologie, supra note 44, art. 14.

169. Liberman c. Tabah, [1990] R.J.Q. 1230 (C.A.).

170. Pépin c. Hôpital du Haut Richelieu, [1983] C.A. 292.

171. Code de déontologie, supra note 44, art. 14. 
manuelle du placenta suivie de la révision utérine manuelle ou de procéder à la réanimation de la femme ou du nouveau-né. ${ }^{172}$

Ce qui signifie que la sage-femme doit avoir la compétence et l'entraînement pour poser ces gestes. L'habileté à pratiquer des manoeuvres de réanimation cardio-respiratoire et à utiliser l'équipement de réanimation ne se conserve que par une pratique régulière et une mise à jour continue. Cependant, l'urgence n'est plus une cause d'exonération si la sage-femme a fait défaut de diagnostiquer correctement la condition de la mère et ou de son enfant, rendant le risque prévisible.

Enfin, avant de cesser d'exercer ses fonctions auprès d'une cliente, la sage-femme doit s'assurer que cette cessation ne lui est pas préjudiciable ${ }^{173}$ et transmettre diligemment les renseignements qu'elle possède au professionnel qui prend la parturiente en charge ${ }^{174}$.

\section{Conclusion}

La légalisation de la profession de sage-femme a amené deux changements importants par rapport à la situation des projets-pilotes. Tout d'abord, la sage-femme obtient le statut d'entrepreneure indépendante. Le CLSC ne répond pas de la faute de la sage-femme puisqu'il n'est lié que par un contrat de services avec la sage-femme alors que le centre hospitalier n'a aucun contrat avec la sage-femme ${ }^{175}$ mais seulement une entente inter-établissements avec le CLSC. En cas d'interventions pluridisciplinaires, une victime aura à démêler tout l'écheveau des soins afin de bien déterminer les responsabilités et leurs auteurs ${ }^{176}$ :

- $\quad$ sage-femme responsable et sage-femme coéquipière;

172. Loi sur les sages-femmes, supra note 1, art. 6 al. 2 .

173. Code de déontologie, supra note 44, art. 17.

174. Ibid., art. 54.

175. LSSSS, supra note 17, art. 236.

176. Hôpital de Chicoutimi c. Battikha, [1997] R.J.Q. 2121 (C.A.). Goupil, supra note 157. 
- $\quad$ CLSC pour ses propres fautes et la faute de ses préposées (responsable des services de sage-femme ${ }^{177}$, infirmières, aides-natales);

- $\quad$ médecin traitant de la parturiente;

- médecin consultant avec suivi conjoint ou non;

- médecin «patron» et résidents du centre hospitalier;

- $\quad$ centre hospitalier pour ses propres fautes et la faute de ses préposés (infirmières, techniciens).

Dans un système de santé publique, est-il juste que la victime ait ce fardeau dans ce qui n'est, au fond, qu'une querelle d'assureurs?

Le deuxième changement important permet à la sage-femme de continuer à suivre, dans certains cas, une grossesse à risque s'il y a eu consultation médicale. Rappelons que la consultation médicale n'est pas obligatoire dans tout suivi de grossesse par une sage-femme, mais seulement dans les cas énumérés au Règlement sur les cas de consultations ou si le bien de la femme l'exige. Devant cette obligation de renseignement renforcée, la sage-femme devra s'assurer du consentement libre et éclairé de la parturiente et documenter son dossier de façon détaillée. Dans l'hypothèse d'un suivi conjoint médecin/sage-femme, la victime devra identifier précisément le professionnel fautif.

La légalisation de la profession de sage-femme est un fait. Il est à espérer que tous les intervenants en périnatalité collaborent efficacement entre eux pour assurer une intégration réussie. Le temps des chicanes inter-professionnelles est terminé. Il faut mettre fin à l'isolement des pratiques. Les professionnels de la périnatalité doivent élaborer des protocoles précis quant au partage des responsabilités en cas de suivi conjoint médecin/sage-femme. Il est important de savoir qui est en charge, qui fait quoi et de partager efficacement l'information. La sécurité de la femme et de son enfant nous impose des obligations de résultat.

177. Houde c. Côté, supra note 60, responsabilité d'un centre hospitalier pour la faute du chef d'un département clinique. 


\begin{abstract}
ANNEXE
La sage-femme pratique avec une philosophie propre à sa profession. Elle travaille toujours selon le regard qu'elle porte sur les femmes et sur
\end{abstract} la maternité en général.

\title{
Philosophie
}

LA PRATIQUE DES SAGES-FEMMES est basée sur le respect de la grossesse et de l'accouchement comme processus physiologiques normaux, porteurs d'une signification profonde dans la vie des femmes.

LES SAGES-FEMMES reconnaissent que l' accouchement et la naissance appartiennent aux femmes et à leur famille. La responsabilité des professionnelles de la santé est d'apporter aux femmes le respect et le soutien dont elles ont besoin pour accoucher avec leur pouvoir, en sécurité et dans la dignité.

LES SAGES-FEMMES respectent la diversité des besoins des femmes et la pluralité des significations personnelles et culturelles que les femmes, leur famille et leur communauté attribuent à la grossesse, à la naissance, et à l'expérience de nouveau parent.

LA PRATIQUE DES SAGES-FEMMES s'exerce dans le cadre d'une relation personnelle et égalitaire, ouverte aux besoins sociaux, culturels et émotifs autant que physiques des femmes. Cette relation se bâtit dans la continuité des soins et des services durant la grossesse, l'accouchement et la période postnatale.

LES SAGES-FEMMES encouragent les femmes à faire des choix quant aux soins et services qu'elles reçoivent et à la manière dont ceux-ci sont prodigués. Elles conçoivent les décisions comme résultant d'un processus où les responsabilités sont partagées entre la femme, sa famille (telle que définie par la femme) et les professionnelles de la santé. Elles reconnaissent que la décision finale appartient à la femme.

LES SAGES-FEMMES respectent le droit des femmes de choisir leur professionnelle de la santé et le lieu de l'accouchement, en accord avec les normes de pratique de l'Ordre des sages-femmes du Québec. Les sages-femmes sont prêtes à assister les femmes dans le lieu d'accouchement de leur choix, incluant le domicile dès que l'assurance professionnelle est en ordre.

LES SAGES-FEMMES considèrent que la promotion de la santé est primordiale dans le cycle de la maternité. Leur pratique se base sur la prévention et inclut un usage judicieux de la technologie.

LES SAGES-FEMMES considèrent que les intérêts de la mère et de son enfant à naître sont liés et compatibles. Elles croient que le meilleur moyen d'assurer le bien-être de la mère et de son bébé est de centrer les soins sur la mère.

LES SAGES-FEMMES encouragent le soutien des familles et de la communauté comme moyens privilégiés de faciliter l'adaptation des nouvelles familles.

Source : Ordre des sages-femmes du Québec, voir en ligne : <http://www.osfq.org/>. 